\title{
SOLUTION OF A GENERAL LINEAR DIFFERENCE EQUATION
}

\author{
S. A. H. RiZVI
}

(Received 26 March 1980)

(Revised 2 September 1980)

\begin{abstract}
A matrix solution and a determinantal solution are obtained for a general linear recurrence relation.
\end{abstract}

A few years back, Brown [1] gave the solution of a three-term linear difference equation

$$
a_{0}(n) u_{n}+a_{1}(n) u_{n-1}+a_{2}(n) u_{n-2}=0, \quad n \geqslant 2,
$$

with $a_{0}(n) \neq 0$ for all $n \geqslant 2$, in terms of certain determinants. In a recent paper, Singh [2] has given the solution for an $(r+1)$-term homogeneous linear difference equation in terms of certain lower Hessenberg determinants, and has also given a matrix solution.

In this note, we obtain a matrix solution as well as a determinantal solution of the non-homogeneous linear difference equation

$$
a_{0}(n) u_{n}+a_{1}(n) u_{n-1}+\cdots+a_{r}(n) u_{n-r}=v(n), \quad n>r,
$$

with $a_{0}(n) \neq 0$ for all $n \geqslant r$. Besides, we establish that the particular solutions appearing in the solution obtained in [2] constitute a linearly independent set under certain conditions.

Following Singh [2], we shall be using the notations:

$a_{t}(n)=0$ if $t$ is a negative integer or a positive integer $>r$.

$p_{k}=\Pi_{h=r}^{k} a_{0}(h)$, empty products being taken to be 1 .

$A_{k}=\left[a_{t-1}(k r+i-1)\right] ; i, j=1,2, \ldots, r ; k=1,2, \cdots$.

$B_{k}=\left[a_{r-(j-i)}(k r+i-1)\right] ; i, j=1,2, \ldots, r ; k=1,2, \cdots$.

$N=[n / r]$, where $[x]$ denotes the integral part of $x ; N^{\prime}=n-N r+1$. 


$$
\begin{aligned}
& A_{(n)}=\left[a_{i-j}(N r+i-1)\right] ; i, j=1,2, \ldots, N^{\prime} . \\
& B_{(n)}=\left[a_{r-(j-i)}(N r+i-1)\right] ; i=1,2, \ldots, N^{\prime} ; j=1,2, \ldots, r . \\
& U_{(r, n)}=\left[u_{r}, u_{r+1}, \ldots, u_{n}\right]^{T} ; U_{(k r,(k+1) r-1)} \equiv U_{k} . \\
& D_{m}^{n}(r, s)=\left|a_{t-j+1}(m+i-1)\right| ; i=1,2, \ldots, n-m+1 ; \\
& \quad j=2-s, 2,3, \ldots, n-m+1, \text { for } n \geqslant m+1 ; \\
& D_{m}^{m}(r, s)=a_{s}(m) ; D_{n+1}^{n}(r, 1)=1 \text { and } D_{r}^{n}(r, s)=D_{s}^{n} . \\
& V_{(r, n)}=[v(r), v(r+1), \ldots, v(n)]^{T} ; V_{(k r,(k+1) r-1)} \equiv V_{k} . \\
& W_{(s, n)}=\left[a_{s}(r), a_{s+1}(r+1), \ldots, a_{s+n-r}(n)\right]^{T} . \\
& \tilde{B}_{k}=A_{k}^{-1} B_{k}, \tilde{V}_{k}=A_{k}^{-1} V_{k} ; k=1,2, \ldots .
\end{aligned}
$$

We shall first obtain a matrix solution of (1). If we put $n=k r, k r+$ $1, \ldots,(k+1) r-1$, in (1), we get the matrix reduction formula

$$
A_{k} U_{k}=V_{k}-B_{k} U_{k-1}, \quad k \geqslant 1,
$$

whence we can easily see that

$$
\begin{gathered}
U_{k}=\left\{(-1)^{k} \prod_{s=k}^{1} \tilde{B}_{s}\right\} U_{0}+\tilde{V}_{k}+\prod_{t=k-1}^{1}(-1)^{k+t}\left(\prod_{s=k}^{t+1} \tilde{B}_{s}\right) \tilde{V}_{t}, k \geqslant 2, \\
U_{1}=\tilde{V}_{1}-\tilde{B}_{1} U_{0} .
\end{gathered}
$$

Therefore, if $n=k r+t, 0 \leqslant t \leqslant r-1$, the general solution of the linear difference equation (1) is given by $u_{k r+t}=$ the $(t+1)$-th element of the matrix $U_{k}$, where $U_{k}$ is defined by (2).

We now obtain the solution of (1) in terms of the determinants $D_{s}^{n}$ and $D_{m}^{n}(r, 1)$. Let $n=r, r+1, \ldots, n$ in (1). Then we get

$$
\begin{aligned}
A_{(r, n)} U_{(r, n)} & =\left(V_{1}-B_{1} U_{0}\right)_{n}, & r \leqslant n \leqslant 2 r-1 \\
& =\left[\begin{array}{l}
V_{1}-B_{1} U_{0} \\
V_{(2 r, n)}
\end{array}\right], & n \geqslant 2 r,
\end{aligned}
$$

where $\left(V_{1}-B_{1} U_{0}\right)_{n}$ denotes the $(n-r+1)$-vector obtained by taking the first $(n-r+1)$ components of $V_{1}-B_{1} U_{0}$, and $A_{(r, n)}=$ the $(n-r+1)$-th leading principal submatrix of $A_{1}$, if $r \leqslant n \leqslant 2 r-1$,

$$
=\left[\begin{array}{cccccc}
A_{1} & O_{r} & \cdots & O_{r} & O_{r} & O_{r, N^{\prime}} \\
B_{2} & A_{2} & \cdots & O_{r} & O_{r} & O_{r, N^{\prime}} \\
O_{r} & B_{3} & \cdots & O_{r} & O_{r} & O_{r, N^{\prime}} \\
\hdashline O_{r} & O_{r} & \cdots & B_{N-1} & A_{N-1} & O_{r, N^{\prime}} \\
O_{N^{\prime}, r} & O_{N^{\prime}, r} & \cdots & O_{N^{\prime}, r} & B_{(n)} & A_{(n)}
\end{array}\right], \quad \text { if } n \geqslant 2 r,
$$

$O_{r, s}$ denoting the null matrix of dimension $r \times s$, with $O_{r, r} \equiv O_{r}$. 
Applying Cramer's rule to the linear non-homogeneous system (3), we get in particular

$$
\left|A_{(r, n)}\right| u_{n}=C_{(r, n)}
$$

where

$$
\begin{aligned}
C_{(r, n)} & =\left|A^{*}{ }_{(r, n)}\left(V_{1}-B_{1} U_{0}\right)_{n}\right|, \quad r \leqslant n \leqslant 2 r-1, \\
& =\left|\begin{array}{cc}
A^{*}{ }_{(r, n)} & V_{1}-B_{1} U_{0} \\
V_{(2 r, n)}
\end{array}\right|, \quad n \geqslant 2 r,
\end{aligned}
$$

$A_{(r, n)}^{*}$ denoting the matrix obtained from $A_{(r, n)}$ by omitting the last column.

Writing $C_{(r, n)}$ as a sum of $(r+1)$ determinants, we obtain

$$
C_{(r, n)}=\left|A_{(r, n)}^{*} V_{(r, n)}\right|-\sum_{s=r}^{1}\left|A^{*}{ }_{(r, n)} W_{(s, n)}\right| u_{r-s}
$$

Now bringing the last column in all these $(r+1)$ determinants to the leading position while retaining the order of the rest of the columns in them, we find that

$$
C_{(r, n)}=(-1)^{n-r+1}\left\{\sum_{s=r}^{1} D_{s}^{n} u_{r-s}-D_{(r, n)}\right\} .
$$

Here $D_{(r, n)} \equiv\left|V_{(r, n)} A_{(r, n)}^{*}\right|$ is obtainable from $D_{s}^{n}$ by replacing its first column by $V_{(r, n)}$, so that, expanding along this column, we get

$$
D_{(r, n)}=\sum_{s=r}^{n}(-1)^{s-r} v(s) p_{s-1} D_{s+1}^{n}(r, 1)
$$

Further

$$
\left|A_{(r, n)}\right|=p_{n} \text {. }
$$

Therefore, from equations (4), (5) and (7), the general solution of (1) is given by

$$
u_{n}=(-1)^{n-r+1} p_{n}^{-1}\left\{\sum_{s=r}^{1} D_{s}^{n} u_{r-s}-D_{(r, n)}\right\}, \quad n \geqslant r
$$

where $D_{(r, n)}$ is given by (6). Taking $v(n)=0, n \geqslant r$, in (6) and (8), we get the following solution for the homogeneous case obtained by Singh [2]:

$$
u_{n}=(-1)^{n-r+1} p_{n}^{-1} \sum_{s=r}^{1} D_{s}^{n} u_{r-s}, \quad n>r .
$$

We now make certain observations regarding the homogeneous case just referred to. In this case, the $r$ particular solutions $(-1)^{n-r+1} p_{n}^{-1} D_{s}^{n}, s=r$, $r-1, \ldots, 1$ (corresponding, respectively, to the initial conditions $u_{r-k}=\delta_{s}^{k}$, 
$k=r, r-1, \ldots, 1$, where $\delta^{\prime}$ is the Kronecker delta) form a linearly independent set of solutions if

$$
a_{r}(h) \neq 0, \quad r \leqslant h<2 r-1 .
$$

This can be seen by observing that a non-trivial linear relation for $n \geqslant r$ between the determinants $D_{s}^{n}$ (as functions of the variable $n>r$ ) implies the existence of a homogeneous system of $r$ equations in $r$ undetermined coefficients with the determinant

$$
E_{r}^{r} \equiv\left|D_{J}^{r+i-1}\right|=0, \quad i, j=1,2, \ldots, r .
$$

But, we have [2]

$$
E_{r}^{r}=\left\{\prod_{n=r}^{2 r-2} p_{n}\right\} q_{2 r-1}
$$

where $q_{k}=\prod_{h=r}^{k} a_{r}(h)$. Since $p_{n} \neq 0$ for $n \geqslant r$ by assumption, $E_{r}^{r} \neq 0$ if (9) holds.

I am grateful to Dr. V. N. Singh for kind help and guidance during the preparation of this paper. I also wish to thank the referees for their helpful suggestions and comments.

\section{References}

[1] A. Brown, "Solution of a linear difference equation", Bull. Austral. Math. Soc. 11 (1974), 325-331.

[2] V. N. Singh, "Solution of a general homogeneous linear difference equation", J. Austral. Math. Soc. B 22 (1980), 53-57.

Department of Mathematics and Astronomy

Lucknow University

Lucknow 226007

India 